\title{
Les minorités d'origine indo-pakistanaise au Kenya, en Tanzanie et en Ouganda
}

Minority groups of Indo-Pakistani origin in Kenya, Tanzania and Uganda

\section{Michel Adam}

\section{(2) OpenEdition}

12 Journals

Édition électronique

URL : http://journals.openedition.org/transcontinentales/788

ISBN : 978-2-7351-1557-0

ISSN : 1775-397X

Éditeur

Editions de la maison des sciences de l'homme

\section{Édition imprimée}

Date de publication : 31 décembre 2010

ISSN : 1950-1684

\section{Référence électronique}

Michel Adam, «Les minorités d'origine indo-pakistanaise au Kenya, en Tanzanie et en Ouganda », Transcontinentales [En ligne], 8/9 | 2010, document 3, mis en ligne le 31 décembre 2010, consulté le 07 septembre 2020. URL : http://journals.openedition.org/transcontinentales/788

Ce document a été généré automatiquement le 7 septembre 2020

Tous droits réservés 


\title{
Les minorités d'origine indo- pakistanaise au Kenya, en Tanzanie et en Ouganda
}

Minority groups of Indo-Pakistani origin in Kenya, Tanzania and Uganda

\author{
Michel Adam
}

1 Étroitement mêlées aux vagues de migrations en provenance de la péninsule Arabique et du golfe Persique, les premières colonies indiennes de l'Afrique orientale sont formées de marchands et participent à la genèse de la civilisation swahilie (du sud de la Somalie au nord du Mozambique). Sollicités par les Britanniques au début du $\mathrm{xx}^{\mathrm{e}}$ siècle, des mouvements migratoires plus importants accompagnent la mise en place de l'ordre colonial, les Indiens étant investis en Afrique dans les fonctions intermédiaires d'artisanat qualifié, de petit commerce, de service et d'auxiliaires administratifs.

2 En croissance rapide (100 000 personnes au début des années 1930, 300000 vers 1950, approchant 400000 personnes en 1962), les minorités d'origine indienne comptent à la veille des indépendances pour près de $20 \%$ de la population urbaine de l'Afrique orientale. Bénéficiant d'une promotion économique rapide, elles s'insèrent dans un cadre socioéconomique gouverné par deux principes régulateurs :

3 - division ethnique et communautaire du travail : la société coloniale est composée de trois ensembles communautaires spécialisés et hiérarchisés. Les Européens se réservent les fonctions d'administration et d'exploitation agro-exportatrice (au Kenya : thé, café, coton, etc.); les Africains sont confinés dans les tâches de production vivrière, de service subalterne et d'auxiliaires de l'agriculture marchande ; les Indiens exercent à la fois les fonctions d'intermédiaire commercial et administratif et de production secondaire artisanale et industrielle ;

4 - complémentarité et non-concurrence intercommunautaire: encadrées dans des activités spécifiques et largement exclusives, les communautés échangent des biens et des services, mais n'entretiennent aucune compétition de caractère économique. Tandis que, au Kenya en particulier, les Indiens sont généralement exclus de 
l'exploitation de la terre, les Africains de leur côté ne sont pas habilités à pratiquer des cultures destinées à l'exportation et demeurent à l'écart du commerce et de l'industrie. Les trois communautés restent socialement et physiquement séparées conformément aux modalités de l'apartheid (système des "réserves ", ségrégation résidentielle en ville, ségrégation scolaire et sociale, stricte endogamie, etc.). Sans approuver toutes les conséquences sociales de l'apartheid, les Indiens s'accommodent de la formule tripartite de division du travail et de séparation communautaire qui évoque la division des castes, encore bien vivante chez eux.

Demeurés en marge de la lutte anticolonialiste et mal aimés de la population africaine, les Indiens subissent aux lendemains des indépendances des réactions xénophobes (expulsion partielle et massacres à Zanzibar, expulsion massive en Ouganda, mesures discriminatoires au Kenya). À l'issue d'une période d'incertitude correspondant à une forte régression démographique, ils préservent néanmoins au fil des années leurs caractéristiques sociales et culturelles tout en consolidant fortement leur emprise économique sur la région.

\section{Caractéristiques principales de la diaspora indienne en Afrique orientale}

Au nombre d'environ 180000 personnes (100 000 au Kenya, 65000 en Tanzanie, 15000 en Ouganda), les minorités d'origine indienne de l'Afrique orientale sont essentiellement regroupées dans les grandes métropoles (Nairobi, Mombasa, Dar es Salaam, Kampala), mais se rencontrent également dans les agglomérations secondaires, et jusque dans les centres ruraux les plus excentrés. Ces minorités se sont, depuis quelques années, partiellement renouvelées par l'arrivée - plus ou moins régulière - de jeunes migrants en provenance de l'Inde. Représentés en majorité par des célibataires diplômés, ces nouveaux arrivants ambitionnent fréquemment un rebond migratoire à destination d'un pays occidental. De manière générale, la population d'origine indienne de l'Afrique orientale n'a pas cessé d'entretenir sa mobilité géographique, renouvelant en permanence la dispersion des familles dans l'environnement mondial anglophone, tant à l'échelle africaine qu'à l'échelle transcontinentale (du Royaume-Uni à l'Amérique du Nord ou à l'Australie).

7 Originaire dans sa grande majorité du nord-ouest du sous-continent (Gujarât, Punjâb, Mahârâshtra, secondairement Kerala ou Sindh pakistanais), la diaspora indienne n'en forme pas moins en Afrique orientale une sorte d'«Inde en réduction ». À peu près toutes les communautés sociales et religieuses existant en Inde y sont représentées, mais dans des proportions qui ne réfléchissent pas exactement la situation de l'ancienne métropole. Tandis que les musulmans - et, à l'intérieur du groupe des musulmans, les chiites - y occupent une part très supérieure à celle de l'Inde (35 à $40 \%$ de musulmans au lieu de $14 \%$ en Inde, $40 \%$ de chiites au lieu de $13 \%$ ), les populations de statut supérieur ou moyen y sont également surreprésentées - tandis que sont absents les «intouchables» et les groupes de très bas statut. Au sein du groupe majoritaire dit localement "hindou» (au sens très large du terme), la part des organisations schismatiques et dissidentes est remarquable : $15 \%$ de jaïns au lieu de $0,3 \%$ en Inde, $8 \%$ de sikhs au lieu de $1,8 \%$.

D'autres minorités comme les chrétiens et les pârsîs figurent également en bonne place parmi les représentants de la diaspora. Cette forte présence des minorités religieuses 
encourage chez les Indiens de l'Afrique orientale une tradition de tolérance qui contribue à la bonne entente entre communautés.

9 Les Indiens sont regroupés en une centaine d'associations communautaires constituées conformément à trois critères de différenciation également actifs en Inde: l'appartenance confessionnelle (tous les sous-groupes hindous étant fédérés dans des Hindu Councils nationaux), la caste (ou la corporation professionnelle qui en tient lieu chez les Sikhs et les musulmans), la région d'origine. Les associations communautaires sont des instances d'entraide et de coopération, de protection sociopolitique, de régulation sociale, d'animation et de sauvegarde culturelle et religieuse. Elles possèdent leurs édifices religieux, leurs centres de loisirs, leurs clubs, parfois leurs écoles, leurs hôpitaux, leurs sociétés de crédit, leurs stations de radio-TV, etc.

\section{La place des Indiens dans les sociétés africaines}

10 Appelés à choisir, au lendemain des indépendances, entre le départ et l'intégration dans le nouvel ensemble sociétal et politique, les Indiens de l'Afrique orientale optèrent pour une voie médiane, plébiscitant l'établissement dans le pays, mais refusant l'intégration complète à laquelle les invitaient les nouvelles autorités. Soucieux de leur côté de ne pas mettre en péril des secteurs d'activités considérés comme vitaux pour la prospérité du pays, les gouvernements est-africains acceptèrent ce compromis. Bien qu'elle eût été malmenée à plusieurs reprises au détriment des communautés immigrées (en particulier à Zanzibar et en Ouganda), la situation nouvelle qui venait ainsi d'être créée devait se prolonger jusqu'à nos jours. Ses caractéristiques peuvent être décrites de la manière suivante :

1. - survivance partielle de la division communautaire et statutaire du travail,

2. - volonté de conservation et d'affirmation de la culture des origines,

3. - faible insertion sociale et politique.

11 En dépit de l'abolition par les nouveaux États africains du cadre juridique de l'ancienne division ethnique $\mathrm{du}$ travail (barrières statutaires, protections corporatistes, ségrégation légale, etc.) et de la mise en compétition des Indiens avec les Africains soucieux de promotion économique et sociale, les Indiens ont maintenu et à certains égards consolidé leur emprise sur les activités de commerce et d'industrie. Ils contrôlent aujourd'hui environ $80 \%$ des branches industrielles (agro-alimentaire, textile, métallurgie, pharmacie, matières plastiques, papeterie et emballage, chimie, etc.), une partie croissante de l'agro-exportation (primeurs et horticulture), du tourisme et de l'hôtellerie, 50 \% des banques et de l'assurance, l'essentiel du commerce de gros et de détail, une fraction très importante des activités immobilières et des professions libérales. Dans les entreprises contrôlées par les Indiens, le recrutement s'opère sur des bases familiales et communautaires, le personnel africain étant, sauf exception, cantonné dans des postes subalternes.

Alors que le cloisonnement professionnel associé aux castes a aujourd'hui perdu son caractère contraignant, la répartition des métiers propres à chaque communauté correspond encore, pour une part, à d'anciennes traditions corporatistes : travail du cuir, orfèvrerie, blanchisserie, métallurgie, textile, banque, etc. Dûs pour l'essentiel à l'inertie des situations acquises (transmission des savoirs et des capitaux), ces continuums technico-professionnels - d'ailleurs fréquemment modernisés et mis au 
goût du jour - n'en contribuent pas moins à la mainmise des Indiens sur certaines branches d'activité.

Socialement, les Indiens demeurent très faiblement intégrés à la société africaine. À l'ancienne ségrégation imposée par la tutelle coloniale s'est substituée une autoségrégation informelle qui laisse peu de place à la mixité sociale et culturelle : quartiers résidentiels séparés, établissements scolaires confessionnels, hôpitaux communautaires, clubs mondains et sportifs, etc.

14 Héritage de prescriptions religieuses et statutaires, l'endogamie communautaire résiste aux tentations de mixité matrimoniale, y compris au sein même de la diaspora. Alors que l'endogamie religieuse (toujours très stricte) suffit déjà à réduire les intermariages entre Indiens et Africains à une petite minorité de musulmans sunnites (le chiisme n'ayant pratiquement aucun adepte en Afrique orientale), l'endogamie ethnique au sens large ne représente aux yeux des Indiens qu'une forme élargie de l'endogamie de caste et paraît du même coup aller de soi - l'endogamie de caste conservant pour la grande majorité sa force dissuasive. Il n'y a donc que fort peu de mariages indoafricains. La logique de l'enfermement communautaire a deux effets complémentaires : elle limite l'imprégnation des cultures africaines chez les représentants de la diaspora sauf l'usage de la langue swahilie généralement adopté par tous les Indiens; elle contribue fortement à la conservation de la culture indienne d'origine (religion, langue, vêtement, alimentation, code social, esthétique, etc.), culture demeurée étrangère et fréquemment indéchiffrable aux yeux des Africains.

La faible implication de la diaspora indienne dans la vie politique de ses pays d'accueil représente un autre aspect de la juxtaposition communautaire propre à l'Afrique orientale. Les Indiens forment des groupes de pression informels, parfois très intriqués dans les cercles du pouvoir. Mais, sauf exception, ils ne participent pas aux débats politiques publics, n'affichent guère d'engagement partisan et ne délèguent que fort peu de représentants dans les instances délibératives locales ou nationales.

\section{Problèmes identitaires et avenir des Indiens en Afrique}

16 À bien des égards toutefois, la situation des Indiens ne correspond pas à la caricature qui en est donnée par les slogans xénophobes, lesquels veulent les faire étrangers à leur pays d'accueil et des émissaires d'intérêts rivaux de celui-ci. Les Indiens d'Afrique de l'Est ont largement rompu leurs attaches avec la patrie de leurs ancêtres. Même si l'Inde demeure un lieu de pèlerinage occasionnel et une référence indépassable pour revitaliser la culture des origines (musique, danse, littérature, cinéma, etc.), elle est devenue familialement, socialement, économiquement et politiquement une contrée étrangère et ne représente en aucun cas un recours migratoire à rebours. Présents en Afrique, pour la plupart, depuis plusieurs générations, détenteurs, dans leur majorité, de la citoyenneté kenyane, oubanguienne ou tanzanienne, les Indiens de l'Afrique orientale sont tout autant des Africains d'origine indienne, Indo-Africains ou AsianAfricans comme ils se dénomment eux-mêmes.

17 Comparable à celle des Indiens d'Afrique du Sud - mais distincte des cas antillais ou indonésien -, la situation identitaire des Indiens d'Afrique de l'Est est originale et à certains égards inédite. Le caractère minoritaire de certaines confessions religieuses - 
justement dominantes en Afrique orientale, comme les ismaéliens, les bohra, les jaïns, les pârsîs, etc. - a contribué à faire de l'attache communautaire transnationale une référence protectrice privilégiée, supérieure à celle des États-nations. C'est ainsi que les ismaéliens, par exemple, trouvent dans les institutions agakhanistes des services correspondant à ceux d'un quasi-État (bancaires, judiciaires, hospitaliers, éducatifs, etc.). De telles attaches transnationales facilitent la mise en place de réseaux industriels, commerciaux et financiers. Indépendamment ou non de tout phénomène d'expansion économique, la dispersion fréquente des familles entretient en permanence l'attache avec d'autres régions du monde et le cosmopolitisme des références. Déjà marquées par l'influence de plusieurs cultures (indienne, anglosaxonne, africaine) et la pluri-appartenance identitaire (indo-communautaire, nationale-africaine), nombreuses par exemple sont les familles de la diaspora disséminées par les contingences des cursus académiques (enfants scolarisés dans des universités anglo-saxonnes) ou par celles de l'émigration secondaire (autre pays africain, Amérique du Nord, Europe, Australie, etc.). L'identité nationale, à laquelle les Africains reprochent aux Indiens de n'être pas attachés - sans qu'ils le soient toujours eux-mêmes davantage qu'à une autre identité régionale ou clanique - n'est pas, cependant, pour les Indiens une valeur négligeable. Mais elle doit composer avec d'autres référents s'inscrivant dans des trajectoires individuelles complexes et, à certains égards, uniques. Le problème des Indiens n'est plus alors de choisir entre plusieurs parcours à la fois extérieurs et intérieurs à leur personne; il est plutôt de découvrir le moyen de faire coexister ces multiples composantes tout en respectant leurs devoirs de citoyen et les lois de leur pays d'accueil.

L'ensemble des caractéristiques culturelles propres aux Indo-Africains en général évoque la description, faite par Arjun Appadurai, des diasporas "post modernes " (Appadurai 2001) ou encore la «fin des territoires » dessinée par Bertrand Badie (Badie 1995). À bien des égards, ces minorités dispersées de par le monde sont des illustrations de la globalisation en marche. Sans attache territoriale exclusive, elles déploient leurs activités à une échelle transcontinentale et communiquent aisément avec leurs correspondants lointains dans un monde sans frontières. Pour autant, elles n'ont pas renoncé à leurs attaches identitaires. La référence à des ressources culturelles en grande partie recomposées ou imaginées - comme c'est le cas, par exemple, des références territoriales des anciennes castes situées dans un passé lointain et abstrait fait revivre les "communautés imaginées » de Benedict Anderson et contribue à la mobilisation de leurs ethnoscapes respectifs (Anderson 1996).

Citoyens de leur pays et citoyens du monde ? C'est à peu près dans ces termes que se présente en Afrique de l'Est une minorité de militants politiques plaidant en faveur de l'intégration. Dépourvus d'appartenance partisane et d'attache politicienne, ils plaident la cause de l'intégration citoyenne (préférence nationale en matière économique, civisme fiscal, ouverture des entreprises à du personnel dirigeant africain, etc.) sans renier la diversité de leurs références culturelles ou de leurs attaches cosmopolites. Représentés par des avocats, des enseignants, des médecins, des commerçants, ils affichent la réalité de leurs liens avec des Africains et donnent parfois l'exemple de la mixité matrimoniale. En dépit du soutien de la presse libérale (Daily Nation de Nairobi, par exemple), leurs engagements, toutefois, rencontrent pour l'heure assez peu d'échos auprès de l'opinion. 


\section{BIBLIOGRAPHIE}

ADAM, M. 2004. «Qui sont les populations d'origine indienne au Kenya », Les Cahiers d'Afrique de l'Est (nouvelle série), 24 : 2-49.

ADAM, M. 2006. «Une minorité microcosmique : les Indo-Kenyans de Nairobi », in H. CHARTONBIGOT et D. RODRIGUEZ-TORRES, Nairobi contemporain. Les paradoxes d'une ville fragmentée. Paris, Karthala (Hommes et sociétés) et Nairobi, IfRA : 285-358.

ADAM, M. (éd.). 2009. L'Afrique indienne. Les minorités d'origine indo-pakistanaise en Afrique orientale. Paris, IFRA-Karthala (Hommes et sociétés).

ANDERSON, B. 1996. L'imaginaire national. Réflexions sur l'origine et l'essor du nationalisme. Paris, La Découverte, (trad. française de Imagined Communities : Reflexions on the Origin and Spread of Nationalism. Londres, Verso, 1991).

APPADURAI, A. 2001. Après le colonialisme. Les conséquences culturelles de la globalisation. Paris, Payot (trad. française de Modernity at Large. Cultural Dimensions of Globalization, Minneapolis, University of Minnesota Press, 1996).

BADIE, B. La fin des territoires. Paris, Fayard, 1995.

BHARATI, A. 1972. The Asians in East Africa. Jayind and Uhuru. Chicago, Nelson Hall Company. GHAI, D. P. et Y. P. GHAI (eds.). 1965. Portrait of a Minority. Asians in East Africa. Oxford, Oxford University Press.

GREGORY, R. G. 1993. Quest for Equality. Asian Politics in East Africa, 1900-1967. New Delhi, Orient Longman.

KAPUR Dromson, N. 2007. From Thelum to Tana. New Delhi, Londres, Penguin Books.

LANDY, Fr. 2003. « Terre des ancêtres et territoire de diaspora : le cas des Sud-Africains d'origine indienne », in Ph. GERVAIS-LAMBONY, Fr. LANDY et S. OLDSFIELD (éd.), Espaces « arcs en ciel ». Territoires et identités en Afrique du Sud et en Inde. Paris, Karthala : 187-206.

MAHARAJ, B. 2003. «Une minorité privilégiée devient indésirable. La diaspora indienne en Afrique », in Ph. GERVAIS-LAmbonY, Fr. LANDY et S. OldSFIELD (éd.), Espaces « arcs en ciel ». Territoires et identités en Afrique du Sud et en Inde. Paris, Karthala : 103-126.

MANGAT, J. S. 1969. A History of the Asians in East Africa, 1886-1945. Oxford, Clarendon Press.

MORRIS, H. S. 1956. «Indians in East Africa : a study in a plural society », British Journal of Sociology, septembre : 194-211.

MoRRIS, H. S.1958. "The divine kinship of the Aga Khan : a study of theocracy in East Africa ", Southwestern Journal of Anthropology, 14(4) : 454-472.

PRUNIER, G. 1990. L'Ouganda et la question indienne. Paris, Éditions Recherches sur les civilisations.

Prunier, G. 1998. « Les communautés indiennes », in Fr. Grignon. et G. Prunier (éd.), Le Kenya contemporain. Paris-Nairobi, Karthala-IFRA : 191-208.

SALVADORI, C. [1983] 1989. Through Open Doors. A View of Asian Cultures in Kenya. Nairobi, Kenway Publications. 
SingaRAVElou, P. 2003. « Les réseaux ethno-culturels et la mondialisation : l'exemple de la diaspora indienne », in Fr. BART et A. LENOBLE-BART (éd.), Afrique des réseaux et mondialisation. Paris, Karthala (Hommes et sociétés) : 17-28.

\section{RÉSUMÉS}

Déjà soumise à l'influence arabe et persane, l'Afrique de l'Est se signale à l'attention des voyageurs par la présence d'importantes colonies d'origine indo-pakistanaise. Attestée depuis plusieurs siècles, l'existence de ces diasporas témoigne du rayonnement de l'Inde à la périphérie de l'océan Indien. Toutefois, la plus grande partie des populations désignées localement sous le nom de Asian peoples est issue d'une immigration récente, résultant de l'élargissement de la sphère impériale britannique à la fin du siècle dernier. Originaires du sud-est du Pakistan (Sindh) et des provinces du nord-ouest et de l'ouest de l'Inde (Punjâb, Râjasthân, Gujarât, Mahârâshtra), ces minorités se sont établies dans les métropoles urbaines ainsi que dans les agglomérations secondaires de l'Afrique orientale. Ayant rapidement assuré leur promotion sociale, elles exercent, à l'échelle locale, nationale et continentale, un rôle économique de premier plan. Leur grande diversité statutaire (survivance du système des castes) et religieuse (musulmans sunnites et chiites, hindous, jains, sikhs, chrétiens, etc.) est entretenue par des stratégies de spécialisation communautaire (commerce de détail et de gros, import-export, industrie, finance, etc.). La pratique persistante de l'endogamie religieuse contribue à les isoler de la population africaine et à faire naître à leur endroit des représentations globalisantes de caractère négatif et xénophobe. Ces réactions de rejet réactivent des sentiments d'appartenance supra-communautaire issus d'une histoire millénaire. Indo-africaines, car néanmoins devenues des composantes essentielles des sociétés est-africaines, mais fortement marquées par la dispersion cosmopolite (MoyenOrient, Europe, Amérique), les diasporas d'origine indienne préfigurent, à l'heure de la mondialisation, de nouvelles identités politiques et culturelles marquées par la multiplicité des appartenances. À ce titre, elles méritent une étude monographique et comparative qui n'avait jamais été engagée en langue française. Se proposant d'éclairer le passé et le présent d'un ensemble de communautés expatriées complexe et multiforme, la présente communication s'appuie sur une recherche conduite depuis 2001 en Afrique orientale sous la direction de Michel Adam et financée par l'Institut français de recherche en Afrique à Nairobi (Adam, éd. 2009).

In addition to being subject to Arab and Persian influences, Eastern Africa is home to large groups of Indo-Pakistani origin. Several centuries old, these groups are evidence of India's presence all around the Indian Ocean. However, most of these groups, known locally as "Asian peoples," are recent immigrants who travelled to the region following the expansion of the British Empire late last century. Originally from southeast Pakistan (the Sindh Province) or northwest and western India (Punjab, Rajasthan, Gujarat and Maharashtra), they moved to urban centres or smaller urban areas in Eastern Africa. After quickly moving up the social ladder, they now play key economic roles on the local, national and continental scale. Their statutory and religious differences (as a result of the caste system and the presence of Sunni and Shiite Muslims, Hindus, Jains, Sikhs, Christians, etc.) are maintained by communitarian specialisation strategies (in retail and wholesale commerce, import-export businesses, industry, finance, etc.). The enduring influence of religious endogamy means they remain isolated from African populations and become the target of negative and xenophobic generalisations. This rejection reactivates supra-communitarian feelings of belonging dating back thousands of years. Nevertheless, the Indian diaspora is now Indo-African and has become an integral part of East African society, albeit affected by international migration (to the Middle East, Europe and America). In today's globalised era, this diaspora is evidence of new political and cultural 
identities marked by multiple belonging. For this reason, they deserve their own monographic and comparative study, never before undertaken in French. The present study examines the past and present of several complex and multiform expatriate communities. It is based on research carried out in East Africa since 2001 directed by Michel Adam and financed by the Institut français de recherche en Afrique (French Institute for Research in Africa) in Nairobi (Adam, ed. 2009).

\section{INDEX}

Thèmes : migration, mobilité

Mots-clés : diaspora indo-pakistanaise, colonie, asian peoples

Index géographique : Afrique orientale, Inde, Pakistan, Kenya, Ouganda, Tanzanie

\section{AUTEUR}

\section{MICHEL ADAM}

Professeur émérite, université François Rabelais de Tours, ancien membre du Conseil scientifique de l'Institut français de recherche en Afrique à Nairobi (ifra-umifre 24) http://www.ifra-nairobi.net/auprès du ministère des Affaires étrangères (1993 à 2004) micheladam@wanadoo.fr 